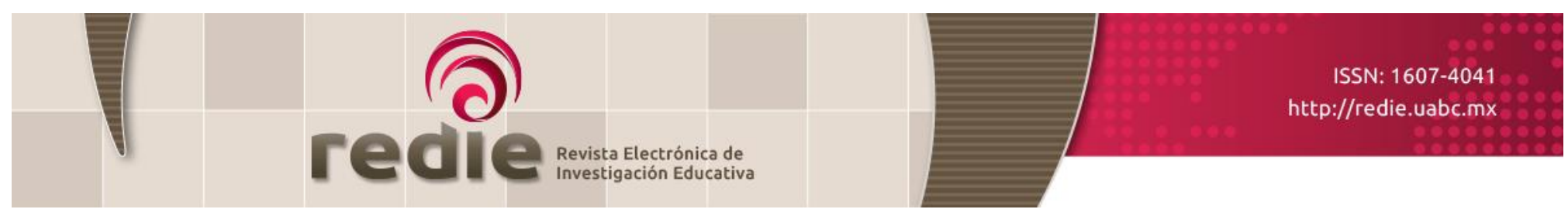

Vol. 20, Núm. 1, 2018

\title{
Representaciones sociales: el significado de la Educación Física para los estudiantes de esa disciplina
}

\author{
Social Representations: the Meaning of "Physical Education" \\ for Students of the subject
}

\author{
Alexis Caniuqueo Vargas (1) alexis.caniuqueo@uautonoma.cl \\ Claudio Hernández-Mosqueira (2) claudio.hernandez@ulagos.cl \\ Angélica Troyano Agredo (3) angelicatroyanoa@gmail.com \\ Daniel Riquelme Uribe (4) daniel.riquelme.uribe@usach.cl \\ Ángelo Vargas (5) angelo.vargas@uol.com.br \\ Rodrigo Vargas Vitoria (6) rvargas@ucm.cl \\ José Fernandes Filho (5) centrodeexcelencia@gmail.com \\ (1) Universidad Autónoma de Chile \\ (2) Universidad de Los Lagos \\ (3) Universidad de San Sebastián \\ (4) Universidad de Santiago \\ (5) Universidad Federal de Río de Janeiro \\ (6) Universidad Católica del Maule \\ (Recibido: 19 de julio de 2016; Aceptado para su publicación: 17 de agosto de 2016)
}

Cómo citar: Caniuqueo, A., Hernández-Mosqueira, C., Troyano, A. Riquelme, D., Vargas, A., Vargas, R. y Fernandes, J. (2018).

Representaciones sociales: el significado de la educación física para los estudiantes de esa disciplina. Revista Electrónica de Investigación Educativa, 20(1), 104-111. https://doi.org/10.24320/redie.2018.20.1.1497

\section{Resumen}

La presente investigación tuvo por objetivo analizar las representaciones sociales de estudiantes chilenos respecto al significado que tiene para ellos la Educación Física, disciplina que estudian. Se utilizó un diseño no experimental, de corte transversal y carácter mixto; la muestra estuvo compuesta por 200 estudiantes de la carrera de Pedagogía en Educación Física de 12 universidades del país, seleccionados bajo un criterio no probabilístico intencionado. Los resultados muestran como palabras definidoras con mayor peso semántico: salud, deporte, motricidad y movimiento. Se concluye que las representaciones sociales de esta especialidad están vinculadas a la salud y bienestar, asociadas al movimiento y estudio de la motricidad humana, otorgando un valor pedagógico a través de elementos como la actividad física, la recreación y el deporte.

Palabras clave: Representaciones sociales, educación física, estudiantes. 


\section{Abstract}

This subject sought to analyze Chilean physical education students' social representations by exploring what physical education means to them. A non-experimental, cross-sectional and mixed-method design was used. The sample was made up of 200 students from the pedagogy in physical education degree in 12 Chilean universities, who were selected under purposive non-probability criteria. The results show that the defining words with the greatest semantic weight are "health", "sport", "motor skills", and "movement". The study concludes that social representations in this field are linked to health and wellbeing, and relate to movement and the study of human motor skills, providing pedagogical value through aspects such as physical activity, recreation, and sport.

Keywords: Social representations, physical education, students.

\section{Introducción}

Las representaciones sociales son definidas como construcciones simbólicas que se originan en la interacción social y se recrean a lo largo del tiempo (Abric, 2001; Banchs, 1986). Son de orden cognitivo, dado que los individuos no son pasivos frente a la información que reciben del grupo, sino que construyen significados y teorías sobre la realidad en una vinculación dialéctica entre lo individual y lo social (Piña y Cuevas, 2004). El concepto de representación social designa una forma de conocimiento específico, el saber de sentido común -cuyos contenidos manifiestan la operación de procesos generativos y funcionales socialmente caracterizados. En sentido más amplio, designa una forma de pensamiento social (Piña y Cuevas, 2004; Rodríguez, 2003).

Las representaciones sociales constituyen un todo estructurado y organizado compuesto por un conjunto de informaciones, creencias, opiniones y actitudes referidas a un objeto o situación y son sociales por ser compartidas por un grupo en común, posibilitando la comprensión del origen de los comportamientos y de las prácticas sociales y, al mismo tiempo, saber qué tipos de elementos pudieran influir en las mismas (León, 2002; Mazzitelli, Aguilar, Guirado, y Olivera, 2009; Piña y Cuevas, 2004; Rodríguez, 2003; Ribeiro y Antunes-Rocha, 2016).

En este sentido, el conocimiento se representa en la Memoria a Largo Plazo (MLP) en una variedad de formas (Lacy y Stark, 2013), pero específicamente en la memoria semántica mediante proposiciones. Dichas proposiciones son unidades mínimas de información almacenada en nuestro cerebro, que corresponde aproximadamente a una idea sobre la realidad que nos circunda (Gagné, 1992; Irish y Piguet, 2013; Pakhomov, Jones y Knopman, 2015).

La proposición es una unidad semántica, no es una frase o una oración. La proposición es lo que se codifica en la memoria. Codificar significa organizar sistemáticamente en la memoria la nueva información, articulándola con la información precedente (Binder, Desai, Graves y Conant, 2009; Hernández-Forte, 2007).

Por otro lado, el utilizar las redes semánticas naturales para desarrollar un estudio de las representaciones sociales se relaciona con la utilización de la memoria explícita, la cual se define como el almacenamiento cerebral de hechos (memoria semántica) y eventos (memoria episódica), por lo cual se expresa conscientemente y es fácil de declarar verbalmente o por escrito (Aguado-Aguilar, 2001; Greenberg y Verfaellie, 2010; Morgado, 2005). En este sentido la memoria explícita resulta del aprendizaje relacional, una forma de aprendizaje complejo que consiste en analizar, comparar y contrastar diferentes tipos de información. Por ello, utilizar como recurso la comprensión de la memoria semántica obedece a que este sistema organiza el conocimiento conceptual mediante representaciones proposicionales, que permiten independizar las expresiones léxicas, gramaticales y sintácticas de las oraciones del lenguaje natural representando su significado en un código semántico (Rodríguez, 2010). 
En el sentido académico, la identidad del docente se vincula a un conjunto de representaciones sociales de la profesión, que pueden permitir reconocerse a sí mismo, identificarse profesionalmente y diferenciarse de otros grupos profesionales (Arbesú, Gutiérrez y Piña, 2008; Butti, 1999; Casado y Calonge, 2000; López, 1996; Machado y Santos, 2015; Mazzitelli et al., 2009;; Mellado y Chaucono, 2015). Esta representación profesional se construye desde el inicio de la carrera y durante el ejercicio de la profesión, constituyendo un cúmulo de representaciones individuales y sociales que otorgan sentido a la tarea en determinadas situaciones (D’andrea y Corral, 2006; Jiménez, 2006; Laudadío, Mazzitelli y Guirado, 2015; Mazzitelli et al., 2009; Mazzitelli y Guirado, 2010; Torres, Maheda y Aranda, 2004).

En el ámbito de la Educación Física en Chile, el perfil epidemiológico de la población nacional y las problemáticas nutricionales de salud evidenciadas a partir de la aplicación del primer Sistema de Medición de la Calidad de la Educación (SIMCE) por parte Agencia de Calidad de la Educación justifica los cambios de contenidos y ejes curriculares propuestos por el Ministerio de Educación (Mineduc) desde el 2012 (Mineduc, 2012; Moreno, Campos y Almonacid, 2012; Salinas, Correa y Vio, 2013).

A partir de los cambios curriculares, los ejes del currículum se centran en habilidades motrices, vida activa y salud, seguridad y juego limpio, situando a la Educación Física como un elemento de cambio y control de los problemas sanitarios del país, con una orientación a problemas de salud evidenciada en el cambio de nombre la de asignatura a Educación Física y Salud. En este sentido, el profesional de la actividad física debe poseer un conjunto de competencias que se hacen necesarias de acuerdo a las necesidades de enseñanza y aprendizaje ( Blázquez-Sánchez, 2001; Cornejo y Matus, 2013; Salinas y Vio, 2003).

Las representaciones sociales de la Educación Física podrían estar orientadas hacia la salud, aunque para los autores de este estudio no fue posible encontrar información de referencia respecto a esta especialidad. El objetivo de este estudio fue analizar las representaciones sociales de la Educación Física en estudiantes de pre-grado de esta especialidad.

\section{Método}

La muestra estuvo compuesta por 136 estudiantes de Pedagogía en Educación Física, chilenos, asistentes al congreso Nacional de Educación Física en Chillán (en 2014), seleccionados bajo un criterio no probabilístico intencionado, pertenecientes a 12 universidades: 5 estatales (42\%) y 7 privadas (58\%). Рara ser incluidos en el estudio los sujetos debían ser alumnos regulares de alguna universidad chilena y cursar entre 10. y 5o. año. Todos los participantes dieron su consentimiento informado, procediendo de acuerdo a la declaración de Helsinki.

Se utilizó un diseño no experimental, de corte transversal, de carácter mixto. Para la recolección de los datos se aplicó una encuesta través de la técnica de redes semánticas naturales de Valdés y Hernández, y Valdés y Reyes (como se cita en Valdez, 1998), la cual consistió en entregar una hoja impresa con la palabra estímulo "Educación Física", acompañada de dos columnas: una correspondía a la palabra definidora y la otra a la jerarquía. Se solicitó a los encuestados anotar en la primera columna un mínimo de diez palabras que definieran al estímulo y que en la segunda jerarquizaran las palabras que dieron como definidoras, de acuerdo a la importancia, relación o cercanía con la palabra estímulo presentada.

Para el análisis se procedió a identificar: 1) el valor J, que corresponde al total de palabras definidoras generadas por los sujetos para definir el estímulo (Educación Física) y es un indicador de la riqueza semántica de la red; 2) el valor M, resultado de la multiplicación de la frecuencia de aparición por la jerarquía obtenida para cada una de las palabras generadas por los sujetos; 3) Conjunto SAM, grupo de 15 palabras definidoras que obtuvieron los mayores valores $M$ totales, y 4) el Valor FMG, indicador en términos de porcentaje de la distancia semántica que hay entre las diferentes palabras definidoras que conforman el conjunto SAM. 


\section{Resultados}

Las representaciones sociales a partir del análisis de las redes semánticas naturales generan una riqueza semántica de 320 conceptos asociados al estímulo Educación física como parte del valor J. Con relación a la representación de la Educación Física, el núcleo de ésta es representado por el concepto Salud, seguido por Deporte con un peso semántico de 44\%, Motricidad 39\% y Movimiento 29\%. Los conceptos Aprendizaje, Enseñanza, Pedagogía y Educación presentan un menor peso semántico, a pesar de ser estudiantes de pedagogía.

Respecto al núcleo semántico de acuerdo al género, mujeres y hombres comparten el mismo concepto y conjunto SAM. Sin embargo, el concepto Deporte presenta un mayor peso semántico en hombres (44\%) que en mujeres (10\%). En el caso del concepto Vida sana el peso semántico es mayor en mujeres (55\%), que en los varones (17\%).

Los conceptos de Educación, Pedagogía y Conocimientos presentan un menor peso semántico dentro del conjunto SAM, tanto en el grupo general como en mujeres y hombres.

Tabla I. Valor m y valor FMG para el conjunto SAM

\begin{tabular}{l|c|c}
\hline Conjunto SAM & Valor M & FMG \% \\
\hline Salud & 877 & 100 \\
Deporte & 373 & 43 \\
Motricidad & 356 & 41 \\
Movimiento & 223 & 25 \\
Recreación & 223 & 25 \\
Aprendizaje & 165 & 19 \\
Valores & 163 & 19 \\
Bienestar & 155 & 18 \\
Cuerpo & 144 & 16 \\
Vida sana & 143 & 16 \\
Enseñanza & 133 & 15 \\
Actividad física & 132 & 15 \\
Educación & 130 & 15 \\
Pedagogía & 130 & 15 \\
Trabajo & 29 & 3 \\
\hline
\end{tabular}

Tabla II. Valores m y fMG para el conjunto sAm por género

\begin{tabular}{lcc|lcc}
\hline \multicolumn{3}{c}{ Mujeres } & & \multicolumn{3}{c}{ Hombres } \\
\hline Conjunto SAM & Valor M & FMG \% & Conjunto SAM & Valor M & FMG \% \\
\hline Salud & 133 & 100 & Salud & 744 & 100 \\
Vida sana & 98 & 74 & Deporte & 275 & 32 \\
Motricidad & 50 & 38 & Motricidad & 306 & 41 \\
Movimiento & 31 & 23 & Movimiento & 192 & 26 \\
Recreación & 26 & 20 & Recreación & 197 & 26 \\
Aprendizaje & 25 & 19 & Aprendizaje & 140 & 19 \\
Cuerpo & 25 & 19 & Valores & 138 & 19 \\
Bienestar & 22 & 17 & Bienestar & 133 & 18 \\
Valores & 21 & 16 & Cuerpo & 126 & 17 \\
Deporte & 17 & 13 & Vida sana & 123 & 17 \\
Enseñanza & 15 & 11 & Enseñanza & 118 & 16 \\
Actividad física & 15 & 11 & Actividad física & 117 & 16 \\
Educación & 15 & 11 & Educación & 115 & 15 \\
Pedagogía & 15 & 11 & Pedagogía & 115 & 15 \\
Trabajo & 10 & 8 & Trabajo & 19 & 3 \\
\hline
\end{tabular}




\section{Discusión y conclusiones}

Las representaciones sociales asociadas al núcleo Salud concuerdan con las nuevas propuestas ministeriales generadas para el actual currículo escolar, y se alinean con las problemáticas de salud reflejadas tanto en el SIMCE de Educación Física y en las políticas de salud pública, necesidad que también manifiestan las propuestas de otros países como parte de la Educación Física (Bracht, 2003). Estas políticas promueven la actividad física, el deporte y la vida sana, elementos centrales de la planificación sanitaria tanto como políticas de prevención como de promoción de calidad de vida.

Los nuevos ejes del currículo escolar en el área de la Educación Física y Salud integran el eje de habilidades motrices, considerado tanto en las representaciones sociales de mujeres como en las de los hombres, ubicándose en tercer lugar de acuerdo con el peso semántico. Las representaciones de valores y deportes podrían asociarse al eje de seguridad y juego limpio.

El bajo peso semántico de los conceptos asociados a Pedagogía y Educación podría obedecer al sentido académico y profesional que adoptan los estudiantes en formación a modo de identificarse o diferenciarse de otros estudiantes de pedagogía. Por otro lado, también podría obedecer a la formación profesional, en cuyo caso las representaciones se han construido desde el inicio de la carrera por la influencia de las representaciones sociales e individuales de los académicos que participan en el proceso, influencia que podría ser mayor por parte de los docentes a cargo del currículo de la especialidad (Bracht, 2003; Cagigal, 2010; Blázquez-Sánchez, 2001).

Los estudiantes de Educación Física de Chile han construido representaciones sociales en función del significado de su especialidad vinculada a la salud y bienestar, asociada al movimiento y al estudio de la motricidad humana, otorgando un valor pedagógico a través de elementos como la actividad física, la recreación y el deporte.

Los resultados obtenidos permiten afirmar que los estudiantes de Educación Física de Chile han construido representaciones sociales en función del significado de su proceso de formación profesional, generando una vinculación de la Educación Física a áreas específicas. En este sentido, los estudiantes plantean que su especialidad está fuertemente vinculada desde el punto de vista conceptual y de aplicación a la salud, lo que es probable que tenga sustento debido a la diversidad de evidencias que se han generado en la investigación en los últimos años y que asocian al ejercicio físico como un agente de estimulación y protección de la salud y la calidad de vida durante todo el ciclo vital.

Por otro lado, los estudiantes asocian la Educación Física al concepto movimiento, lo que también se asocia a la influencia de su proceso formativo, debido a la gran cantidad de estudios que le dan al movimiento un rol central en el proceso del desarrollo humano, no sólo desde el punto de vista físico, sino como un potente factor del desarrollo y protección de los fenómenos cognitivos que nos hacen humanos.

Respecto al acercamiento del concepto "motricidad", obedece principalmente a las corrientes que plantean a esta disciplina como una de las encargadas del estudio del movimiento en el contexto de la Educación Física, y cuyo desafío es contribuir al desarrollo de las potencialidades del organismo humano para explorar su propia corporalidad y su entorno a través del movimiento.

Por último, cabe destacar la importancia de continuar el estudio de la representaciones sociales de la Educación Física, pues permitirían desarrollar y promover acciones para fortalecer las nuevas áreas de desarrollo que la Educación Física integra ante los avances en investigación básica y aplicada, que la relacionan con beneficios y mecanismos protectores no sólo en la población infantil y juvenil, sino en todo el ciclo vital. 


\section{Referencias}

Abric, J. C. (2001). Metodología de recolección de las representaciones sociales. Prácticas sociales y representaciones sociales. México: Ediciones Coyoacán.

Aguado-Aguilar, L. (2001). Aprendizaje y memoria. Rev. Neurol., 32(04), 373-381.

Arbesú , M., Gutiérrez, S. y Piña, J. (2008). Representaciones sociales de los profesores de la UAM-X sobre la evaluación de la docencia e investigación. Reencuentro, 53, 85-96.

Banchs, M. (1986). Concepto de representaciones sociales. Análisis comparativo. Revista Costarricense de Psicología, 8(9), 27-40.

Binder, J., Desai, R., Graves, W. y Conant, L. (2009). Where is the semantic system? A critical review and meta-analysis of 120 functional neuroimaging studies. Cerebral Cortex, 19(12), 2767-2796.

doi:10.1093/cercor/bhp055

Blázquez-Sánchez, D. (2001). La educación física: España: Inde.

Bracht, V. (2003). La educación física en Argentina y en Brasil: identidad, desafíos y perspectivas. Argentina: Ediciones Al Margen.

Butti, F. (1999). Representaciones sociales de los maestros y fracaso escolar. Hacia una propuesta metodológica. Reuniones de Comunicaciones Científicas y Tecnológicas. Ciencias Sociales. Universidad Nacional del Nordeste, Argentina.

Cagigal, J. (2010). ¿ La educación física, ciencia? Educación física y deporte, 6(2-3), 49-58.

Casado, E. y Calonge, S. (2000). Representaciones sociales y educación. Venezuela: Comisión de Estudios de Postgrado, Facultad de Humanidades y Educación, Universidad Central de Venezuela.

Cornejo, M. y Matus, C. (2013). Educación Física en Chile. Revista Electrónica Actividad Física y Ciencias, $5(1)$.

D'andrea, A. y Corral, N. (2006). Representaciones sociales de formadores sobre el éxito y el fracaso académico y el buen y el mal estudiante. Actas de la XIII Reunión de Comunicaciones Científicas y Tecnológicas. Universidad Nacional del Nordeste, Argentina. Recuperado de http://www.unne.edu.ar/unnevieja/Web/cyt/cyt2006/09-Educacion/2006-D-007.pdf

Gagné, E. D. (1992). La psicología cognitiva del aprendizaje escolar. España: Visor.

Greenberg, D. y Verfaellie, M. (2010). Interdependence of episodic and semantic memory: evidence from neuropsychology. Journal of the International Neuropsychological Society, 16(5), 748-753.

doi:10.1017/S1355617710000676

Hernández-Forte, V. (2007). Mapas conceptuales: la gestión del conocimiento en la didáctica. España: Alfaomega.

Irish, M. y Piguet, O. (2013). The pivotal role of semantic memory in remembering the past and imagining the future. Frontiers in Behavioral Neuroscience, 7(27), 1-11. doi:10.3389/fnbeh.2013.00027

Jiménez, R. (2006). Motivación, trato de igualdad, comportamientos de disciplina y estilos de vida saludables en estudiantes de educación Física en Secundaria. (Tesis Doctoral). Universidad de Extremadura, España. 
Lacy, J. y Stark, C. (2013). The neuroscience of memory: implications for the courtroom. Nature reviews. Neuroscience, 14(9), 649-658. doi:10.1038/пгп3563

Laudadío, J., Mazzitelli, C. y Guirado, A. (2015). Representaciones de docentes de Ciencias Naturales: Punto de Partida para la Reflexión de la Práctica. Actualidades Investigativas en Educación, 15(3), 159-181.

León, M. (2002). Representaciones sociales: actitudes, creencias, comunicación y creencia social. En F. Morales, A. Kornblit, D. Páez y D. Asún (Coords.), Psicología social (pp. 367-385). Argentina: Prentice Hall Pearson Education.

López, F. (1996). Representaciones sociales y formación de profesores. El caso de la UAS. Revista Mexicana de Investigación Educativa, 1(2), 391-407.

Machado, L. y Santos, J. (2015). Escola organizada em ciclos: as representações sociais de professores considerados bem-sucedidos. Ensaio: Avaliação e Políticas Públicas em Educação, 23(89), 843-868. doi:10.1590/S0104-40362015000400003

Mazzitelli, C., Aguilar, S., Guirado, A. y Olivera, A. (2009). Representaciones sociales de los profesores sobre la docencia: contenido y estructura. Revista Educación, Lectura y Sociedad, 6(6), 265-290.

Mazzitelli, C. y Guirado, A. (2010). La enseñanza y el aprendizaje de las ciencias. Estudios de las representaciones sociales de docentes y futuros docentes en Ciencias. Argentina: FFHA-UNSJ.

Mellado, M. y Chaucono, J. (2015). Creencias Pedagógicas del Profesorado de una Escuela Rural en el Contexto Mapuche. Actualidades Investigativas en Educación, 15(3), 316-334.

Ministerio de Educación. (2012). Informe de Resultados SIMCE Educación Física 8o. Básico 2012 para docentes y directivos. Recuperado de http://archivos.agenciaeducacion.cl/biblioteca digital historica/resultados/2012/ire8b edfisica 2012.pdf

Moreno, A., Campos, M. y Almonacid, A. (2012). Las funciones de la educación física escolar: una mirada centrada en la justicia social y la reconstrucción del conocimiento. Estudios pedagógicos, 38(Especial), 1326. doi:10.4067/S0718-07052012000400002

Morgado, I. (2005). Psicobiología del aprendizaje y la memoria: fundamentos y avances recientes. Rev Neurol, 40(5), 289-297.

Pakhomov, S., Jones, D. y Knopman, D. (2015). Language Networks Associated with Computerized Semantic Indices. Neurolmage, 104, 125-137. doi:10.1016/j.neuroimage.2014.10.008

Piña, J. y Cuevas, Y. (2004). La teoría de las representaciones sociales: Su uso en la investigación educativa en México. Perfiles educativos, 26(105-106), 102-124.

Ribeiro, L. y Antunes-Rocha, M. (2016). História, abordagens, métodos e perspectivas da teoria das representações sociais. Psicologia \& Sociedade, 28(2), 407-409. doi:10.1590/1807-03102016v28n2p407

Rodríguez, R. (2010). Herramientas informáticas para la representación del conocimiento. Subjetividad y procesos cognitivos, 14(2), 217-232.

Rodríguez, T. (2003). El debate de las representaciones sociales en la psicología social. Relaciones Estudios de historia y sociedad, 24(93), 51-80.

Salinas, J., Correa, F. y Vio, F. (2013). Marco normativo para promover una alimentación saludable en escuelas básicas de Chile. Revista Chilena de Nutrición, 40(3), 274-282.

doi:10.4067/S0717-75182013000300010 
Salinas, J. y Vio, F. (2003). Promoción de salud y actividad física en Chile: política prioritaria. Revista Panamericana de Salud Pública, 14(4), 281-288.

Torres, T., Maheda, M. y Aranda, C. (2004). Representaciones sociales sobre el psicólogo: investigación cualitativa en el ámbito de la formación de profesionales de la salud. Revista de Educación y Desarrollo, 2, 29-42.

Valdez, J. L. (1998). Las redes semánticas naturales, usos y aplicaciones en psicología social. México: Universidad Autónoma del Estado de México. 